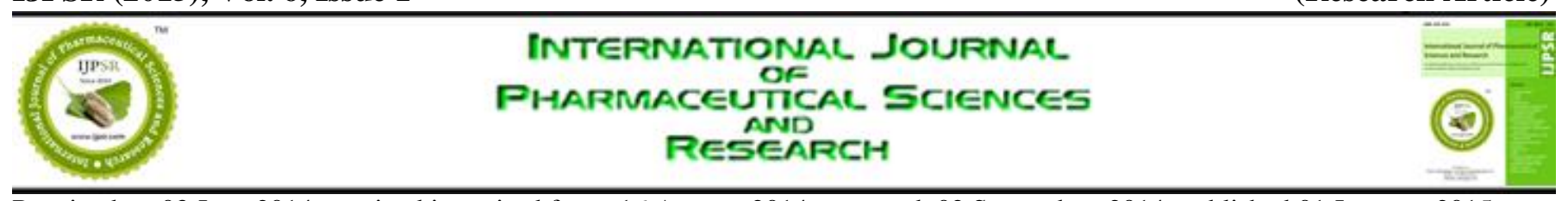

Received on 03 June 2014; received in revised form, 16 August, 2014; accepted, 02 September, 2014; published 01 January, 2015

\title{
EFFECT OF HYDRO-ALCOHOLIC ROOT EXTRACT OF PLUMBAGO ZEYLANICA L ALONE AND ITS COMBINATION WITH AQUEOUS LEAF EXTRACT OF CAMELLIA SINENSIS ON RESERPINE INDUCED PARKINSONISM
}

Sibi P. Ittiyavirah* and R. Ruby

Division of Pharmacology, Department of Pharmaceutical Sciences, Mahatma Gandhi University, Cheruvandoor campus, Ettumanoor P.O., Kottayam, Kerala, India.

Keywords:

Catalepsy, Reserpine,

Parkinsonism, Syndopa,

Plumbagozeylanica, Camellia sinensis, stepping test, open field test

Correspondence to Author:

Dr. Sibi P. Ittiyavirah

Assistant Professor

Division of Pharmacology,

Dept. of Pharmaceutical Sciences,

Mahatma Gandhi University,

Cheruvandoor Campus,

Ettumanoor P.O., Kottayam, Kerala,

India

E-mail: sibitho@gmail.com
ABSTRACT: Aim: To investigate the antiparkinsonism activity of hydro alcoholic root extract of $P$. zeylanica $\mathrm{L}$ (PZE) alone and its combination with aqueous extract of $C$. sinensis leaves (AECS) in reserpine induced model. Materials and methods: Parkinsonism (PD) was induced by sub cutaneous administration of reserpine (1 $\mathrm{mg} / \mathrm{kg}$ ) for 5 days. The extracts/drugs being tested were administered orally (p.o) 60 min prior to the administration of the reserpine for five days. Behavior parameters were analyzed using catalepsy, open field and stepping test. Result: Repeated administration of reserpine $(1 \mathrm{mg} / \mathrm{kg}$; s.c) for a period of 5 days significantly increased catalepsy and decreased the locomotor activity as compared to normal animal. All the groups ie L-dopa + carbidopa (syndopa), PZE alone and its combination with $C$. sinensis showed significant $(\mathrm{P}<0.001)$ reduction in catalepsy, akinesia and increase in locomotor performance. Conclusion: It is concluded that $P$. zeylanica alone and its combination with $C$. sinensis have a protective effect against PD, while bi-herbal extracts showed more significant protective effect. Hence may offer a safer therapeutic approach to the treatment of PD and drug induced dyskinesia.
INTRODUCTION: Parkinson's disease (PD) is an idiopathic, slowly progressive, and chronic neurodegenerative disorder characterized by the selective and progressive loss of the dopaminergic (DAergic) neurons in the substantia nigra pars compacta. The degeneration of dopaminergic neurons results in threshold reduction of approximately $80 \%$ dopamine in the striatum, which lead to the emergence of neuromuscular executive dysfunction, learning problems and mood disorders. ${ }^{1}$ PD has four cardinal features: bradykinesia (slowness and poverty of movement), muscular rigidity, resting tremor and impairment of postural balance leading to disturbances of gait and falling. ${ }^{1-2}$

\begin{tabular}{|l|c|}
\hline QUICK RESPONSE CODE & $\begin{array}{c}\text { DOI: } \\
\text { DOI link: } \text { http://dx.doi.org/10.13040/IJPSR.0975-8232.6(1).485-90 }\end{array}$ \\
\hline
\end{tabular}

The protein abnormalities, environmental toxins, genetic factors, mitochondrial dysfunction, free radical mediated cell death, oxidative stress, neuroinflammation, and cellular inclusions augment the process of selective loss of nigral

DAergic neurons leading to depletion of DA in striatum. ${ }^{3}$ Therefore, the compounds that have properties, such as mitochondrial function enhancement and antioxidant ability may provide the neuroprotective effect. ${ }^{4}$ The pharmacotherapy of PD was limited mostly to symptomatic treatments none of them prevent disease from progressing. ${ }^{2}$ Levodopa has been considered the gold standard drug therapy for Parkinson's disease, but it is limited only to relief symptoms and long term use may cause serious side effects that include involuntary movements (dyskinesia). The side effects of allopathic medicines for PD are highly alarming hence the current research is now focusing on herbal medicines and alternative 
systems of medicine. In addition to several synthetic agents modulating various pathways, certain plant constituents like polyphenols, flavonoids also exhibited some activity against experimentally induced PD. ${ }^{4}$ More over herbal medicines are used to treat PD in ancient medical systems in Asian countries such as India, China, Japan and Korea based on their own anecdotal or experience-based theories. Mucuna pruriens commonly known as velvet beans or cow itch are used in case of spasms associated with Parkinsonism. ${ }^{5}$ Viciafaba (Broad beans) is an herb used for PD and possess higher degree of antiparkinsonism activity compared with L-dopa. ${ }^{6}$

The current study envisages in evaluating the antiparkinsonian effect of hydro alcoholic root extract of Plumbago zeylanica alone and its combination with Camellia sinensis in reserpine model of PD. The studies suggest that free radicals have a key role in neurodegenerative disorders, including Parkinsonism. Free radicals induce lipid peroxidation that leads to neuronal death. This may be due to reduced level of antioxidant such as glutathione that may lead Parkinsonism patient more vulnerable to oxidative stress. ${ }^{7} P$. Zeylanic ${ }^{8}$ and $C$. Sinensis ${ }^{9}$ have potent antioxidant activity; which may reduce the degeneration of neurons associated with PD. These plants contain compounds classified into alkaloids, flavonoid, polyphenols, catechol, terpenes which are the most promising candidate for the treatment of PD. ${ }^{4}$

The root of $P$. zeylanica contains several bioactive constituent like L-dopa, plumbagin, droseron, chitranone, triterpinoid, anthraquinone. ${ }^{10}$ L-dopa present in the herbal drugs reported to provide antiparkinsonism activity without producing drug induced dyskinesia, which is the main side effect of synthetic drugs. ${ }^{5}$ The dried root extract of $P$. zeylanica may ameliorate Parkinsonism without developing side effects. $P$. zeylanica shown to have a number of actions in the central nervous system (CNS) including stimulatory ${ }^{11}$ and nootropic action ${ }^{12}$. Neuroprotective mechanism ascribed to $P$. zeylanica in these studies may also claim to have beneficial effect in PD.

L-dopa is inactive by itself because it does not cross blood brain barrier, but is the immediate precursor of dopamine. L-dopa is decarboxylated to dopamine by the enzymes mono amine oxidase and catechol- o-methyl transferase (COMT). About 1$2 \%$ of orally administered L-dopa crosses the blood brain barrier, is taken up by the surviving dopaminergic neurons, converted to dopamine, which is stored and released.

In modern practice L-dopa is administered with peripheral decarboxylase inhibitors which prevent peripheral decarboxylation of L-dopa and increase the availability of dopamine in the brain. This not only reduces the dose but also the peripheral side effects of L-dopa. ${ }^{13}$ Vast epidemiology data indicated that green tea consumption reduces the occurrence of neurodegenerative disorders such as Parkinsonism and Alzheimer's disease. ${ }^{14}$ Various studies suggested that EGCG may inhibit COMTcatalyzed methylation of endogenous and exogenous compounds. ${ }^{15}$

We assumed that $C$. sinensis may prevent the metabolism of dopamine present in the $P$. zeylanica root extract by inhibiting the enzyme COMT and allows maximum L-dopa to reach the CNS. COMT inhibition may also preserve dopamine formed in the striatum. The synergistic action of $C$. sinensis extract may reduce the dose and peripheral side effect of L-dopa.

\section{MATERIALS AND METHODS: Animals:}

Healthy adult male Wistar albino rats, weighting $150-220 \mathrm{~g}$ obtained from the registered animal house of University College of Pharmacy (UCP), Cheruvandoor campus, Mahathma Gandhi University, Kottayam, Kerala, India and College of Veterinary and Animal Sciences, Mannuthy, Thrissur, India were used for the study.

The study protocol was approved by the Institutional Animal Ethical Committee (IAEC) of UCP bearing IAEC no: [012/MPH/UCP/CVR/13]. All the animals were housed in polypropylene cages, maintained under standard husbandry conditions (12h light and dark cycles, room temperature $\left(27 \pm 2^{\circ} \mathrm{c}\right)$ and $45-55 \%$ relative humidity. The rats were provided with standard pellet diet and water ad libitum throughout the course of the study. 


\section{Drugs:}

Reserpine (Sigma Aldrich, St. Louis, U.S.A), Syndopa (L-dopa+C-dopa). P. zeylanica roots were collected from Madhuvanam botanical garden; Calicut, India and $C$. sinensis leaves were collected from Munnar, Idukki, India during the month of March, 2013. The plants were taxonomically identified and authenticated by Dr. Pandurangann, Scientist F \& Head, Plant systematics and evolutionary science division, Jawaharlal Nehru Tropical Botanical Garden and Research Institute, Palode, Trivandrum, Kerala.

\section{Statistical analysis:}

The statistical analysis was performed using one way analysis of variance (ANOVA) followed by Dunnett's multiple comparison test. Comparisons were made between haloperidol group and test/standard groups. P-values $<0.05$ was considered statistically significant. The statistical analysis was done by using Graph pad prism version no: 6.0 .

\section{Reserpine model of PD:}

Reserpine induces depletion of central catecholamine stores. The sedative effect can be observed in rats shortly after injection, followed by signs of eyelid ptosis, hypokinesia, rigidity, catatonia, and immobility. These phenomena can be antagonized by dopamine agonists. The behavioral parameters including the motor activity were assessed after continuous treatment of Reserpine for a period of 5 days at a concentration of $1 \mathrm{mg} / \mathrm{kg}$.

The animals were divided into five groups, each consisting of six rats. Group-I served as sham and received $0.1 \%$ acetic acid p.o, Group-II received reserpine diluted in $0.1 \%$ glacial acetic acid for five consecutive days at a concentration of $1 \mathrm{mg} / \mathrm{kg}$ s.c. Group III received Syndopa p.o at the dose of $10 \mathrm{mg} / \mathrm{kg}$ on the first day, 60minutes prior to first injection of Reserpine and daily for another five days. Groups IV received PZE alone at the dose of $100 \mathrm{mg} / \mathrm{kg}$ p.o, on the first day, 60min prior to first injection of reserpine and daily for another five days of treatment. Groups V received combination of PZE and AECS, on the first day, 30 minutes prior to first injection of Reserpine and daily for another five days. At the end of experimental period (after five days of treatment) ie on $5^{\text {th }}$ day of the experiment, behavioral studies were performed to understand motor skill abnormalities. ${ }^{16,17,18}$

\section{Behavioral parameters:}

Spontaneous locomotor behavior and abnormal involuntary movements (open field apparatus):

Open field apparatus consist of squares $(61 \times 61)$ were used for the study. Blue lines were drawn on the floor with a marker. The lines divided floor into sixteen squares. A central square was drawn on the middle of open field. The rats were centrally placed in the open field apparatus and were allowed to walk without restraint inside the area for $5 \mathrm{~min}$ and following behavioral aspects were noted:

i). Ambulation: this was measured in terms of the number of squares crossed by the animal;

ii). Rearing frequency: partial or total elevation on to hind limbs;

iii).Self grooming: number of times animal groomed facial region, and licked /washed/ scratched various part of the body;

iv). Activity in Central Square: number of central squares crossed by the animal. Two consecutive days animals were exposed to the apparatus for habituation. The open field was cleaned with a 5\% water-alcohol solution before behavioral testing to eradicate possible bias due to smells left by previous rats.

\section{Akinesia (stepping test):}

Akinesia means loss or impairment of voluntary activity. The level of akinesia was assessed by stepping test. The rat were allowed to stand its forelimbs and allowed to move while its hind limbs were lifted and the number of steps taken with both forelimbs was recorded for 30s.

\section{Catalepsy:}

The catalepsy was assessed by placing the animal's forepaws on a horizontal bar positioned at $9 \mathrm{~cm}$ above the bench surface. The duration of catalepsy, which was defined as an immobile posture, keeping both forepaws on the bar, was measured up to a maximum of $180 \mathrm{~s}$. 


\section{RESULTS:}

\section{Preliminary phytochemical analysis:}

The preliminary phytochemical studies were performed for testing different phytochemical constituents present in hydro-alcoholic root extract of $P$. zeylanica $\mathrm{L}$ and aqueous extract of $C$. sinensisleaves. The observation indicates the presence ofalkaloids, phenolic compounds, flavonoids, phytosterols and terpenoids in both PZE and AECS leaves. In addition to this PZE showed the presence of tannins and proteins Table 1.

TABLE: 1.CHEMICAL CONSTITUENTS PRESENT IN PZE AND AECS

\begin{tabular}{cccc}
\hline Sl. & Test & PZE & AECS \\
No & & & \\
\hline 1. & Alkaloids & + & ++ \\
2. & Carbohydrates & + & + \\
3. & Glycosides & - & - \\
4. & Phytosterols & + & - \\
5. & Fixed oils\& Fats & - & - \\
6. & Saponins & - & - \\
7. & Phenolic compounds & + & + \\
8. & Flavonoids & ++ & ++ \\
9. & Proteins and & + & + \\
10. & aminoaids & & \\
11. & Tannins & + & - \\
12. & Terpenoids & ++ & + \\
\hline
\end{tabular}

+ Indicate presence, - Indicate absence

\section{Catalepsy test:}

The duration of catalepsy was increased in Reserpine treated group when compared to control group. Syndopa, PZE alone and its combination with AECS treated groups sowed decrease in duration of catalepsy as compared to Reserpine treated group. The bi-herbal extract $(\mathrm{P}<0.001)$ has more significant effect than PZE alone $(\mathrm{P}<0.01)$ and it was almost similar to standard group Figure1.

\section{Open field test:}

The open field test was done in order to determine the effect of administration of P. Zeylanica and its combination with C.sinensis upon spontaneous locomotor activity. Locomotor parameters were significantly enhanced in Syndopa, PZE alone and bi-herbal extracts treated group when compared with Reserpine group. The combination of extract has more significant activity on ambulation $(\mathrm{P}<0.01)$, rearing $(\mathrm{P}<0.001)$, grooming $(\mathrm{P}<0.05)$ and activity in centre $(\mathrm{P}<0.05)$ as compared to $\mathrm{PZE}$ alone Figure 2.

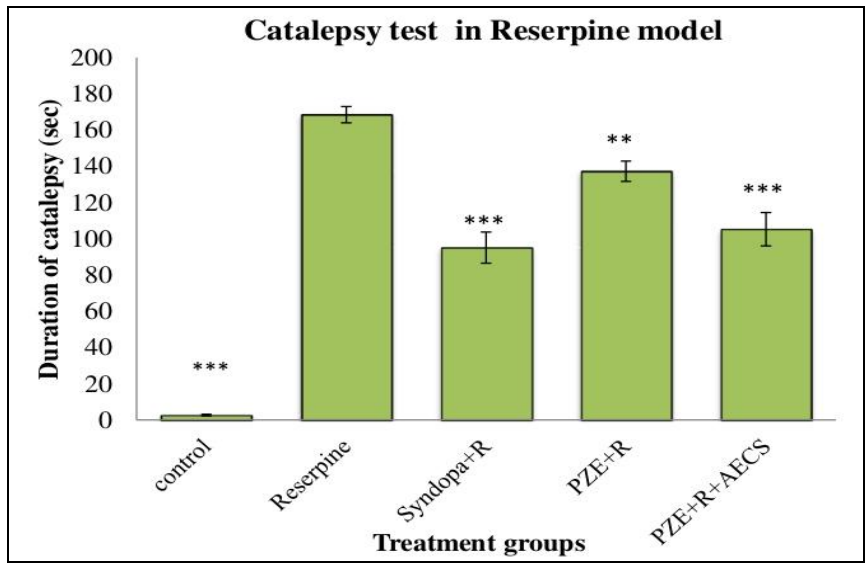

FIGURE 1: EFFECT OF $P$. ZEYLANICA AND ITS COMBINATION WITH $C$. SINENSIS ON RESERPINE INDUCED CATALEPSY TEST. * represents $\mathrm{P}<0.05$, ** $\mathrm{P}<0.01, * * * \mathrm{P}<0.001$ as compared to reserpine treated group.

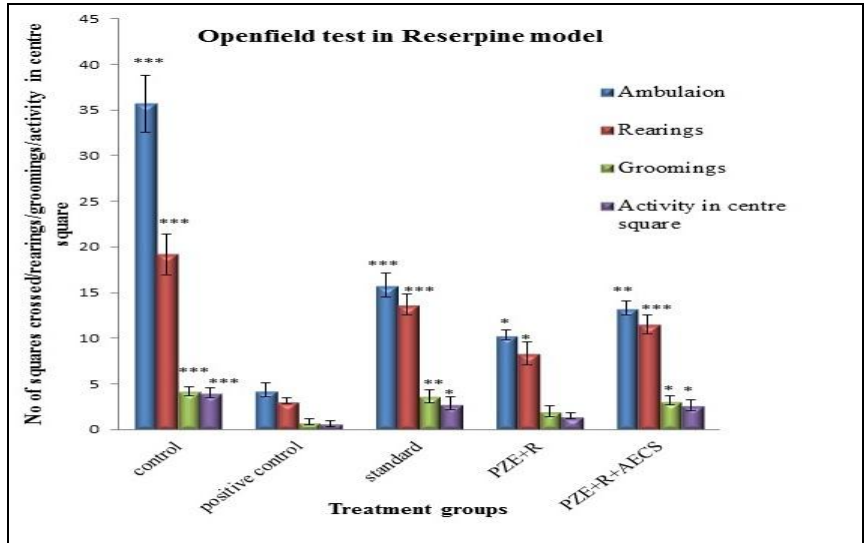

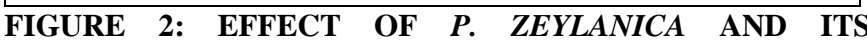
COMBINATION WITH $C$. SINENSIS ON THE TOTAL NUMBER OF LINE CROSSING, REARINGS, GROOMING AND CENTRE SQUARE ENTRY IN MALE ALBINO WISTAR RATS IN RESERPINE MODEL. * represents $\mathrm{P}<0.05$, $* * \mathrm{P}<0.01$, *** $\mathrm{P}<0.001$ as compared to reserpine treated group.

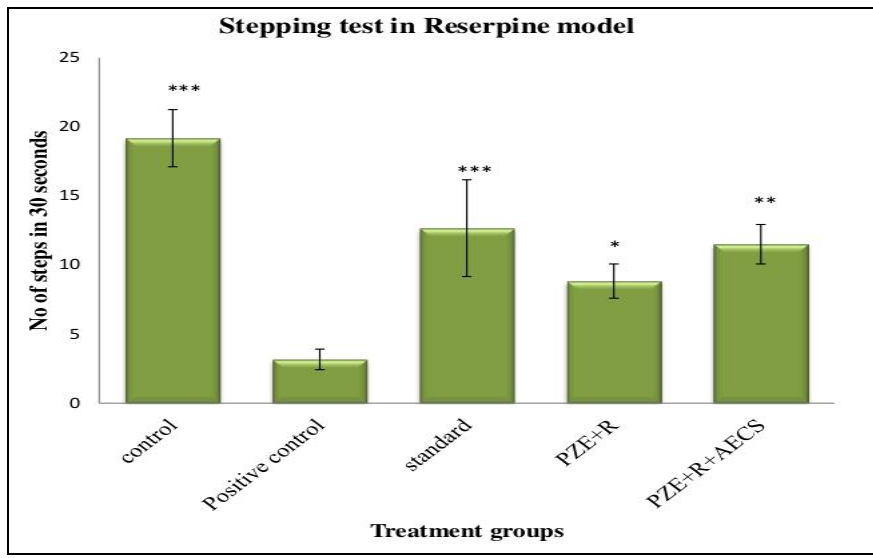

FIGURE 3: EFFECT OF $P$. ZEYLANICA AND ITS COMBINATION WITH $C$. SINENSIS ON STEPPING TEST IN RESERPINE MODEL. * represents $\mathrm{P}<0.05$, ** $\mathrm{P}<0.01, * * * \mathrm{P}<0.001$ as compared to reserpine treated group. 


\section{Stepping Test:}

Hydro-alcoholic extract of P.zeylanica and aqueous extract of C.sinensiswere administered orally to male wistar albino rats and number of steps in 30 seconds was measured. The number of steps in 30 seconds was significantly enhanced on $5^{\text {th }}$ day in Syndopa $(\mathrm{P}<0.001)$, PZE alone $(\mathrm{P}<0.05)$ and its combination with AECS $(\mathrm{P}<0.01)$ treated groups as compared to Reserpine induced Parkinsonism group Figure 3.

DISCUSSION: Reserpine, an alkaloid extracted from the roots of an Indian plant, Rauwolfia serpentina, blocks the ability of aminergic transmitter vesicles to take up and store biogenic amines probably by interfering with an uptake mechanism that depends on $\mathrm{Mg}^{2+}$ and ATP. ${ }^{19}$ In reserpine induced model of $\mathrm{PD}$, catecholamine stores were depleted and motor syndrome characterized by decreased initiation and speed of voluntary movements, rigidity, and a hunched posture was observed.

These symptoms bear many similarities to those seen in Parkinson's disease and are ameliorated by dopaminergic anti-parkinsonian drugs. Thus, the reserpine-treated rodent provides a useful model of Parkinson's disease. ${ }^{20}$ The animals exhibited a debilitating phenotype that was confirmed by behavior analysis like catalepsy, open field and stepping test. The study revealed bradykinesia and increased muscular rigidity in rats exposed to repetitive administration of reserpine Figure 1- 3.

Repeated administration of reserpine ( $1 \mathrm{mg} / \mathrm{kg}$; s.c) for a period of 5 days significantly increased catalepsy and decreased the locomotor activity as compared to normal animal. All the groups ie Ldopa + carbidopa (syndopa), PZE alone and its combination with $C$. sinensis showed significant $(\mathrm{P}<0.001)$ reduction in catalepsy, akinesia and increase in locomotor performance.

The mechanism due to amelioration take place may attribute to one or more pharmacological mechanism. Protective effects against reserpine induced behavioral changes suggest that these plant extracts has influence on aminergic pathway. Reserpine binds tightly to adrenergic vesicles in central and peripheral adrenergic neurons and remains bound for a prolonged period of time. The interaction inhibits the vesicular catecholamine transporter that facilitates the vesicular storage. Catecholamine leaks into the cytoplasm, where they are metabolized through the interneuronal monoamine oxidase, and lead to the formation of 3 , 4- dihydroxy phenyl acetic acid (DOPAC) and hydrogen peroxide.

In presence of ferrous ion, $\mathrm{H}_{2} \mathrm{O}_{2}$ undergoes spontaneous conversion, forming a hydroxyl free radical, causes oxidative stress and degeneration of neuron. ${ }^{21}$ Phytochemical screening of these plants revealed the presence of tannins and phenolic compounds, alkaloids, carbohydrates, and saponins. Literature surveys indicate that flavonoids, alkaloids and phenolic compounds possess antioxidant activity.

The antiparkinsonism effect of PZE and AECS are produced possibly by acting as a free radical scavenger or by the presence of natural L-dopa in $P$. zeylanica roots or by inhibition of COMT by EGCG in green tea extract leading to increase in dopaminergic neurotransmission and decrease in DA metabolites thereby decreasing the formation of ROS. These findings suggested a possible protective role of plant extracts in oxidative stressinduced neurodegeneration and may be beneficial adjuvant or an alternative agent in the treatment or prevention of Parkinsonism and related disorders.

CONCLUSION: It is concluded that $P$. zeylanica alone and its combination with $C$. sinensis have a protective effect against reserpine induced PD, while bi-herbal extracts exhibited more significant activity than single administration. Hence may offer a safer therapeutic approach to the treatment of Parkinson's disease. Being a neuroprotectant it could be used as an effective adjunct to L-dopa for the treatment of neuroleptic-induced extrapyramidal side effects (management of drug induced dyskinesia). These may be valuable for identifying lead compounds for anti-parkinsonism drugs, keeping in mind the side effects of presently used drugs. The standardization of the extracts, identification and isolation of active principles along with pharmacological and mechanism based 
studies of these principles may be the future aspect for further detail studies.

Still further human studies are needed to prove the safety and efficacy of long term administration of extract of $P$. zeylanica alone and its combination with $C$. sinensis. In the light of observations made, it may be envisaged that $P$. zeylanica alone and its combination with $C$. sinensis can be used as a potential adjuvant in the treatment of neurodegenerative disorders.

\section{REFERENCES:}

1. Parkinson J. An essay on the shaking palsy. J Neuropsychiatry ClinNeurosci 2002; 14(2):223-236.

2. Laurence LB, Keith LP. Goodman and Gillman are manual of pharmacology and therapeutics. The McGraw-Hill Publication, New Delhi, 2008.

3. Isacson $\mathrm{O}$. Models of repair mechanisms for future treatment modalities of Parkinson's disease. Brain Res Bull 2002; 57:839-846.

4. Ju-Xian S, Stephen C, Tzi-Bun N, Caivin KL, George PH, Leungc, et al. Anti-Parkinsonian drug discovery from herbal medicine: what have we got from neurotoxic model. J Ethnopharmacol 2012; 139:698-707.

5. Maheswaria T, Vijayrajaa D, Kundhavai Natchiyara R, Kalaivania K, Rajasankar S, Tamilselvama K, Manivasagama T. Synergistic neuropreventive effect of Withania somnifera root powder and Mucuna pruriens seed powder in Parkinsonic mice model. $J$ Herbal Medicine and Toxicology 2010; 4(2):63-69.

6. Kempster PA, Bogetic Z, Secombe JW, Bappl SC, Dmartin H, Balazs NDH Motor effects of broad bean (Viciafaba) in Parkinsonism disease: single dose studies. Asia pacific J Clin Nutr 1993; 2:85-89.

7. Cooke E, Dizdaroglu M, Lunec J. Oxidative DNA damage: mechanisms, mutation, and disease. Food Chemistry 2003; 17:1195-1214.

8. Tilak JC, Adhikari S, Devasagayay TP. Antioxidant property of Plumbagozeylanica, anIndian medicinal Plant and its active ingredientPlumbagin. Redox Report 2004; 9:219-227.

9. Chia-Fang $\mathrm{T}$, Yu-Wen $\mathrm{H}$, Hung-Chih $\mathrm{T}$, Chun-Fa H, Cheng-Chieh $\mathrm{Y}$. The in vivo antioxidant and antifibrotic properties of green tea (Camellia sinensis, Theaceae). Food Chemistry 2013; 136:1337-1344.

10. RamyaKuber B, Santh Rani Thaakur. Herbs containing LDopa: An update. Ancient Science of life 2007; 27(1):5055.

11. Bopaiah CP, Pradhan N. Central nervous system stimulatory action from the root extract of Plumbagozeylanica in rats. Phytother Res 2001; 15(2):153-156.

12. Vineet mittal, Sharma SK, Pawanjalwal, Anil hooda, Morl J. Plumbago zeylanica roots: a potential source for improvement of learning and memory. IntJPharm BiolSci 2010; 6(2):1-6.

13. Goodman and Gillman. Treatment of Central Nervous System Degenerative Disorders. In: Laurence L. Brunton .The pharmacological basis of therapeutics. 12th ed. San Diego, California: The McGraw-Hill Companies.2012.p. 336-345.

14. Feng-Jiao L, Hong-Fang J, Liang S. A meta-analysis of tea drinking and risk of Parkinson's disease. The Scientific World Journal 2012; 12:1-6.

15. Hong L, Xiaofeng M, Chung SY. Enzymology of methylation of tea catechins and inhibition of catechol-omethyltransferase by (-)-epigallo catechin gallate. Drug Metabolism and Disposition 2003; 31(5):572-579.

16. Jose Luis Calderon, Rodrigo Bolanos. Behavioral analysis of the reserpine induced motor changes in a Parkinsonian mouse model. Revist Neuropsicologia, Neuropsiquiatria y Neurociencias 2011; 11:49-61.

17. Valeria SF, Jose R, Anderson HF, Leaoa, Andre M. Medeiros, Thieza $\mathrm{G}$ et al. Repeated treatment with a low dose of reserpine as a progressive model of Parkinson's disease. Behavioural Brain Research 2012; 300:1-10.

18. FabiolaTrevizol, Dalila M, Benvegnu, Raquel CS, Barcelos, Camila S, Pase et al. Comparative study between two animal models of extrapyramidal movement disorders: Prevention and reversion by pecan nut shell aqueous extract. Behavioural Brain Research 2011; 22:13-18.

19. Benowits NL. Antihypertensive agents. In: Katzung, B.G. (Ed.), Basic \& Clinical Pharmacology, ninth ed. McGrawHill Press, New York, 2004.

20. Di Marzo V, Hill MP, Bisogno T, Crossman AR, Brotchie JM. Enhanced levels of endogenous cannabinoids in the globuspallidus are associated with a reduction in movement in an animal model of Parkinson's disease. The Scientific World Journal 2000; 14:1432-1438.

21. Li SW, Lin TS, Minteer S, Burke WJ. 3, 4Dihydroxyphenylacetaldehyde and hydrogen peroxide generate a hydroxyl radical: possible role in Parkinson's disease pathogenesis. Mol. Brain Res 2001; 93:1-7.

\section{How to cite this article}

Ittiyavirah SP and Ruby R: Effect of Hydro-Alcoholic Root Extract of Plumbago Zeylanica L Alone and Its Combination with Aqueous Leaf Extract of Camellia Sinensis on Reserpine Induced Parkinsonism. Int J Pharm Sci Res 2015; 6(1): 485-90.doi: 10.13040/IJPSR.0975-8232.6 (1).485-90.

All $\odot 2013$ are reserved by International Journal of Pharmaceutical Sciences and Research. This Journal licensed under a Creative Commons Attribution-NonCommercial-ShareAlike 3.0 Unported License.

This article can be downloaded to ANDROID OS based mobile. Scan QR Code using Code/Bar Scanner from your mobile. (Scanners are available on Google Playstore) 